\title{
Corrigendum: Real-space imaging of interfacial water with submolecular resolution
}

Jing Guo, Xiangzhi Meng, Ji Chen, Jinbo Peng, Jiming Sheng, Xin-Zheng Li, Limei Xu, Jun-Ren Shi, Enge Wang and Ying Jiang

Nature Materials 13, 184-189 (2014); published online 5 January 2014; corrected after print 21 December 2016.

In the version of this Article originally published, the title of ref. 1 was incorrect and should have read: 'The interaction of water with solid surfaces: fundamental aspects revisited'. This was corrected on 21 December 2016. 